\title{
TBT OPTICS AND IMPEDANCE MEASUREMENTS AT THE FERMILAB MAIN INJECTOR*
}

\author{
Y. Alexahin, E. Gianfelice-Wendt, Fermilab, Batavia, IL 60510, USA
}

\section{Abstract}

The Fourier analysis of Turn by Turn (TBT) data provides valuable information about the machine linear and non-linear optics. This technique introduced first at Fermilab in 2006 for correcting the Tevatron linear coupling, has been now extended to the Main Injector with the aim of a better understanding of the beam dynamics, in particular in view of a substantial beam intensity increase in the frame of the laboratory neutrino program.

\section{INTRODUCTION}

The recent upgrade of the Main Injector (MI) Beam Position Monitor (BPM) system allows the use of Turn by Turn (TBT) techniques for this machine. We use the Fourier transform of the TBT oscillations resulting from a single kick to evaluate the twiss function values at the BPMs location. Other methods, as Model Independent Analysis or Independent Component Analysis, may be used but finally all these methods suffer from the fact that the invariant amplitude, which is relevant for the computation of the $\beta$ function and of the linear coupling, must be computed by resorting to a machine model and from the fact that the amplitude of the coherent oscillations decreases more or less quickly limiting the phase measurement resolution too. On this last respect the use of a socalled AC dipole is of course more convenient, but while not any machine has a $\mathrm{AC}$ dipole, every machine has at least an injection kicker.

\section{OPTICS}

The MI is a rapid cycling multipurpose accelerator delivering $p$ beams for fixed target experiments and $\bar{p}$ and $\nu$ production as well as $\bar{p}$ and $p$ for the Tevatron collider. The results here presented have been obtained by analyzing the TBT data following vertical kicks ${ }^{1}$ at injection energy, namely $8.94 \mathrm{GeV}$. The tune feed forward loop was switched off and the Time Line Generator, which controls the machine events, was kept rigorously constant so to avoid temperature depending energy drifts. The MI is $3319.42 \mathrm{~m}$ long and is equipped with totally 208 BPMs. The large aperture BPMs, which are not yet included in the TBT reading software, have been excluded from our analysis together with those where the beam closed orbit has large nominal offsets. There are left therefore 101 BPMs measuring the beam horizontal position and 98 measuring

\footnotetext{
* Work supported by Fermi Research Alliance LLC. Under DE-AC0207CH11359 with the U.S. DOE.

${ }^{1}$ the (vertical) injection kicker is the only kicker available in the MI
}

the vertical one. In TBT modus each BPM acquires 2048 turns.

We have taken 2 sets of measurements with a total of $0.25 \times 10^{12}$, and $1.5 \times 10^{12}$ particles in 30 bunches respectively. Fig. 1 shows the TBT data at two locations where nominally $\beta_{x}=56 \mathrm{~m}$ and $\beta_{z}=59 \mathrm{~m}$ for one of the $N=0.25 \times 10^{12}$ shots.

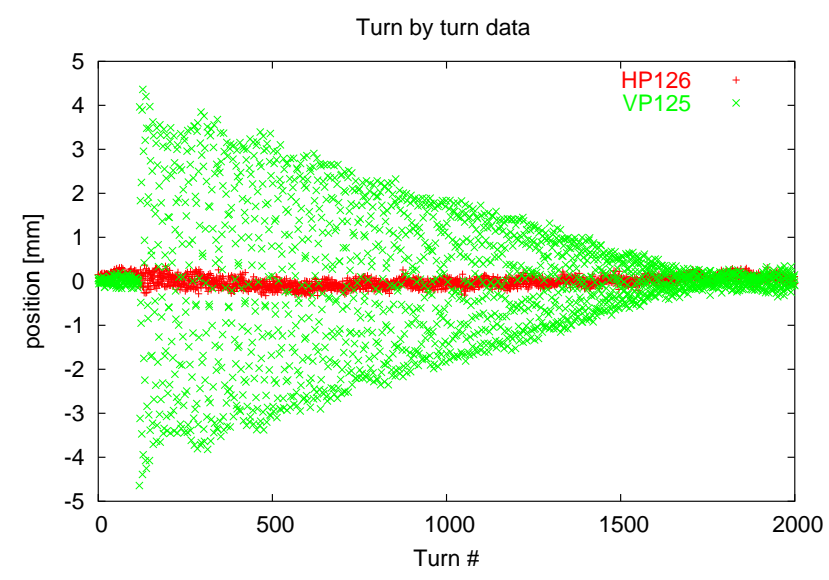

Figure 1: TBT data for $N=0.25 \times 10^{12}$.

The coupling of the vertical oscillation into the horizontal plane is very weak. The poorness of the horizontal data does not allow a meaningful optics measurement in that plane.

Fig. 2 shows the measured vertical $\beta$ function at the BPMs location (averaged over 6 measurements with $N=0.25 \times 10^{12}$ ) as well as the design one (MAD). The error bars are the statistical errors. The corresponding $\beta$ beating is shown in Fig. 3. A Fourier analysis of the $\beta$-beating (see Fig. 4) does not reveal a clear peak at the expected harmonic, namely 42 for $Q_{y}=25.4$ and 93 sampling points, but the largest deviations can be fitted piecewise by $2 Q_{y}$ waves and therefore should be true $\beta$-beating.

Fig. 5 shows the phase advance deviation from the design one. There are no large localized gradient errors.

\section{DEPENDENCE OF PHASE ADVANCE ON BEAM CURRENT}

Measuring the dependence of the phase advance upon beam current one can evaluate the effective reactive impedance of the machine and localize the sources.

The MI vacuum chamber is made out of stainless steel and it is approximately elliptical with a full width of 12.3 $\mathrm{cm}$ and a full height of $5.31 \mathrm{~cm}$. Effects due to the vacuum chamber are therefore expected to be larger in the vertical plane. Fig. 6 and Fig. 7 show the change of phase advance 


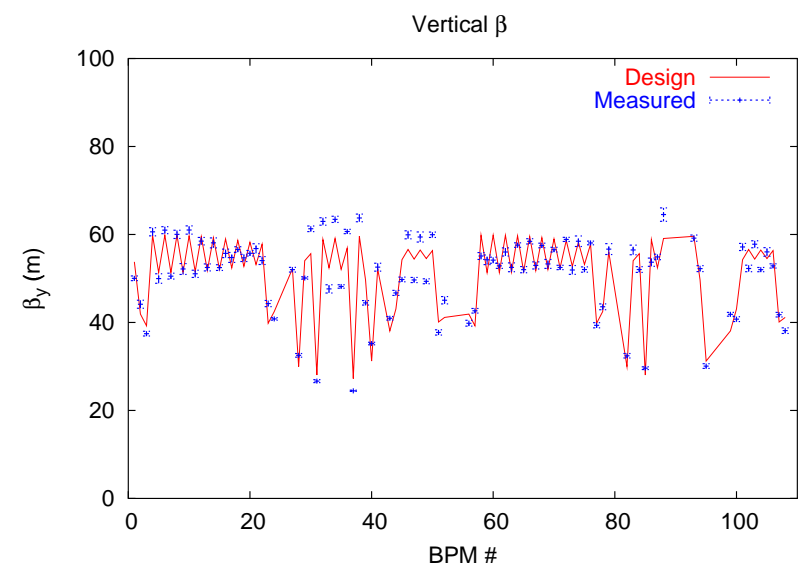

Figure 2: Vertical $\beta$.

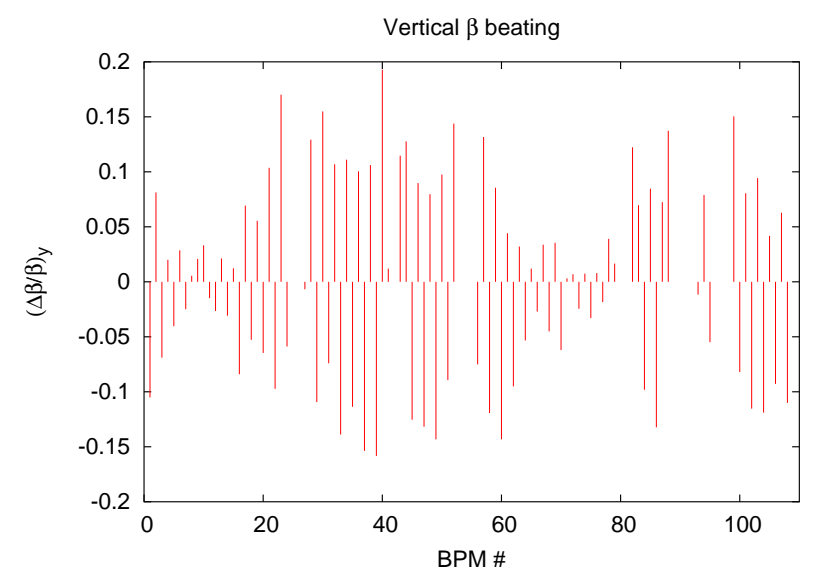

Figure 3: Vertical $\beta$ beating.

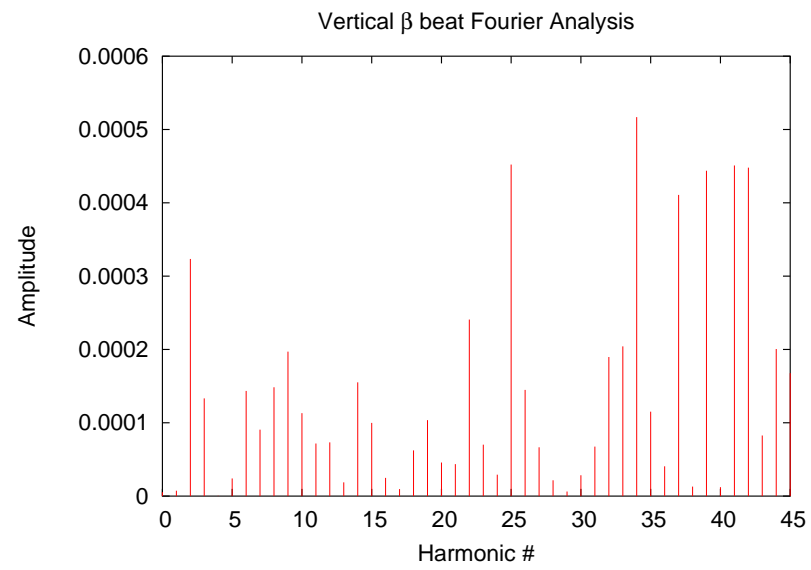

Figure 4: Vertical $\beta$-beating Fourier spectrum.

along the machine in the horizontal and vertical plane respectively.

Unlike the horizontal case, the vertical one show a clear dependence upon beam current. There are no evident steps, the phase advance difference just increases monotonically along the ring. This result indicates a distributed reactive impedance related to the vacuum chamber. We fit linearly

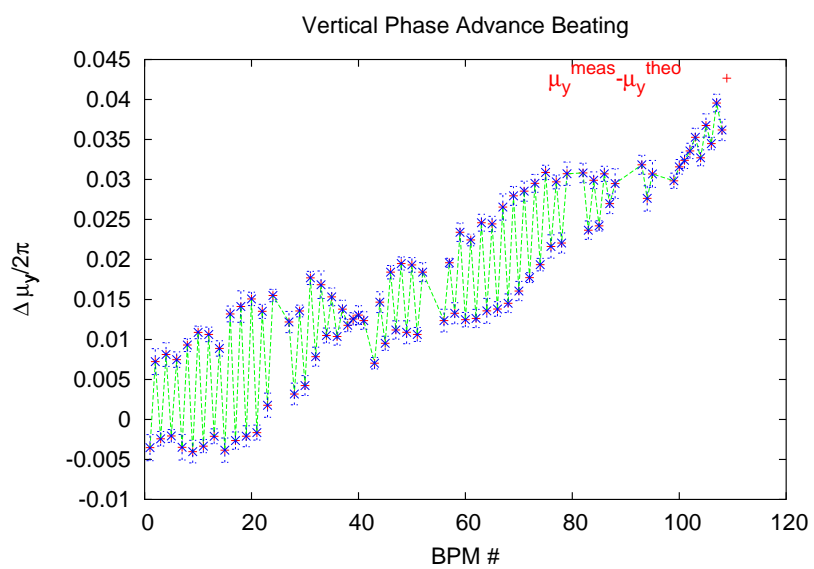

Figure 5: Vertical phase advance beating.

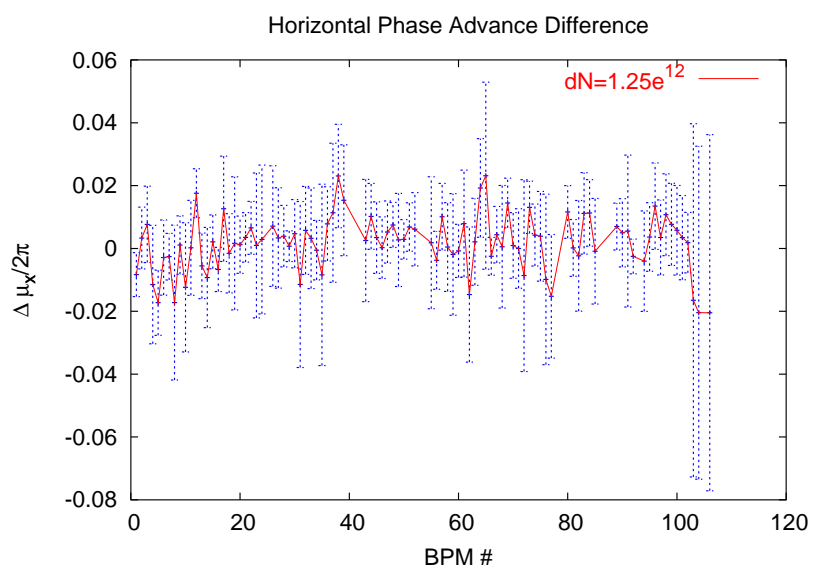

Figure 6: Horizontal phase advance difference for $d N=1.25 \times 10^{12}$.

all data and get

$$
\frac{\Delta \mu_{y}}{2 \pi}(s)=-0.169 \times 10^{-5} \mathrm{~m}^{-1} \times s
$$

We can estimate the effective vertical impedance from the expression [1]

$$
Z_{T}^{e f f}=\frac{2 \sigma_{\ell} E \beta^{2}}{e \sqrt{\pi} R<\beta_{y}>} \frac{\Delta \mu}{\Delta I_{b}}
$$

where $E$ is the beam energy, $\beta=v / c$ and $R$ is the machine mean radius.

Due to the large bunch length, the factor relating phase advance slope and impedance is for hadron machines in general much larger than in lepton machines: the same impedance produces a much smaller effect on the phase advance. This method is therefore for hadron machines not as sensitive as for lepton ones.

For the MI with $<\beta_{y}>=28 \mathrm{~m}, R=528.3 \mathrm{~m}$ and, at injection energy, $\sigma_{\ell}=0.75 \mathrm{~m}$, Eq. 1 becomes

$$
Z_{T}^{e f f}=0.506 \times 10^{6} \mathrm{~V} \mathrm{~m}^{-1} \frac{\Delta \mu}{\Delta I_{b}}
$$




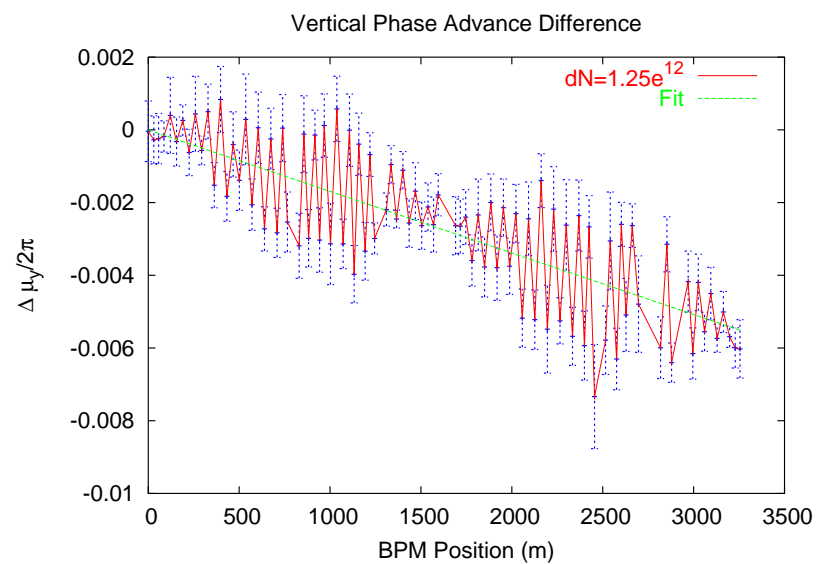

Figure 7: Vertical phase advance difference for $d N=1.25 \times 10^{12}$.

From our fit we get $\Delta Q_{y}=-0.0056 \pm 0.0012$ (in good agreement with the difference of the average tunes computed from the Fourier spectrum, namely -0.0057) that is $\Delta \mu_{y}(L) / \Delta I_{b}=-58.68 \mathrm{rad} \mathrm{A}^{-1}$ which gives $Z^{T}=29.4 \mathrm{M} \Omega$ $\mathrm{m}^{-1}$. We can compare our measured tune shift with the theoretical estimation of the space charge coherent tune shift [2], namely

$$
\Delta Q_{y}^{c o h}=-2.16 \times 10^{-3} \frac{N_{b}}{\tau_{\ell}}-7.53 \times 10^{-6} N_{b} m
$$

where $m$ is the number of bunches, $\tau_{\ell}$ is the bunch length in ns and $N_{b}$ is the number of particles per bunch in units of $10^{10}$. In our case Eq. 2 gives $\Delta Q_{y}^{c o h}=-0.0027$.

\section{ENERGY LOSS DEPENDENCE ON BEAM CURRENT}

The measurement of the dependence upon current of the closed orbit at the BPM locations of non-vanishing dispersion allows to compute the the loss factor $K_{L}$, defined as the energy loss per unit charge squared, which is related to the resistive part of the effective longitudinal impedance. The MI is a nominally flat ring and therefore we have to resort on the horizontal BPMs measurement. The horizontal BPMs TBT data have been averaged to get the closed orbit. For the high current case the TBT position at those locations where the dispersion is non vanishing, shows an oscillation at the synchrotron motion frequency $\left(Q_{s} \simeq 0.0079\right)$, in addition to a slower oscillation very likely due to the dipole magnets ripple [3]. To mitigate their effect we have fitted and subtracted such oscillations before making the average. The result of such data smoothing is shown in Fig. 8 for one of the $N=1.5 \times 10^{12}$ case.

The difference between the high and low current case is shown in Fig. 9. The scatter between measurements is quite large resulting in a relatively large statistical error.

Within the errors, there is no measurable effect and at most we can give an upper limit for the loss factor based

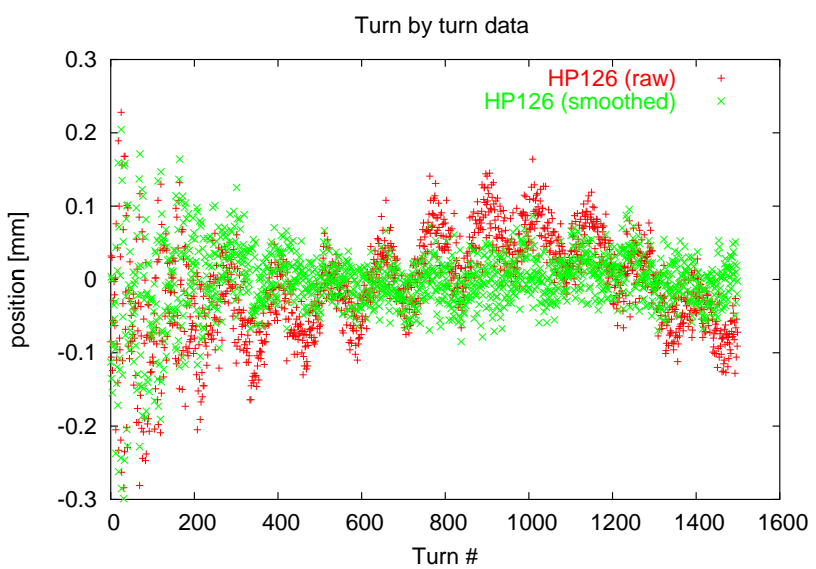

Figure 8: Horizontal TBT for a $N=1.5 \times 10^{12}$ case.

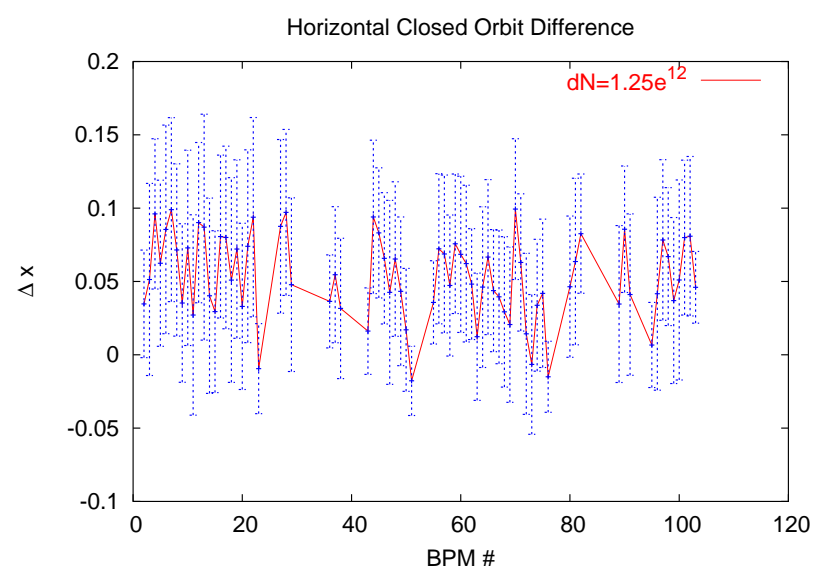

Figure 9: Horizontal closed orbit difference for $d N=1.25 \times 10^{12}$.

on the statistical error, that is [4]

$$
K_{L}=\frac{E f_{r e v}}{e D_{x}} \frac{\Delta x_{c o}}{\Delta I_{b}}<60 \mathrm{~V} \mathrm{p} \mathrm{C}^{-1}
$$

everywhere along the ring.

\section{ACKNOWLEDGMENTS}

The support of R. Zwaska and in particular of D. Capista in the data acquisition is warmly acknowledged.

\section{REFERENCES}

[1] A. W. Chao,"Physics of Collective Beam Instabilities in High Energy Accelerators”, John Wiley \& Sons, New York, 1993.

[2] M. A. Martens and K. Y. Ng, "Impedance and Instability Threshold Estimates in the Main Injector", Fermilab Main Injector Note MI-0103, 1994.

[3] D. Capista, Private Communication.

[4] D. Brandt et al., "Measurement of Impedance Distributions and Instability Thresholds in LEP", PAC'95, Dallas, May 1995, p. 570. 